\title{
Exploring the Use of the Mobile Phone
}

\author{
Nadine Ozkan \\ CSIRO / CMIS \\ Currently: Lanterna Magica Inc,.433 St-Joseph west, \# 20, Montreal, QUE, Canada H2V \\ $2 P 3$ \\ email: nadine.ozkan@lanternamagica.com
}

\begin{abstract}
The work reported here provides insight into the practice of mobile communication. Interviews with experienced mobile phone users were conducted on specific instances of communications they placed, and the corpus gathered was analysed from the following three perspectives: why was a mobile phone used rather than another communication device; what was the intent or the goal of the communication, and, when appropriate, why was text messaging chosen over voice communication.

Results reveal the various punctual reasons that motivate the use of the mobile phone (such as its functionality, its cost, its ease of access), as well as the type of communications placed from a mobile phone. These reasons and communication types all concur to make this device the support of fluid and flexible social interactions and coordination. As to text messaging, it is considered as a medium in its own right, whose use is largely governed by emerging social conventions.
\end{abstract}

Key words: mobile communication, user practices, technology appropriation

\section{INTRODUCTION: RESEARCH QUESTION}

Mobile phones have gained extraordinary popularity since their introduction a few years ago. For example, at the time of writing this paper, an Ericsson advertisement claims that some 200 million SMS messages are sent around the world every day. Mobile communication can truly be described as a social phenomenon. Surprisingly however, mobile communication has not been much studied in the literature. The work 
presented here addresses this gap. The objective is to gather an understanding of the current usage patterns of the mobile phone: What constitutes mobile communication? What factors account for the choice of mobile communication as opposed to stationary communication? What habits and conventions emerge in the community of mobile users, and are re-enacted by this community? In addition, given that mobile phones today allow communication either by voice or text, we also must address the question of the factors motivating mobile phone users' choice between these two channels.

In our investigations, we have chosen to focus specifically on mobile phone usage for communication of a personal nature, i.e., exclusive of workrelated communication. This is motivated by two reasons. The first is that, while much work has been devoted to distance communication and collaboration in work settings, mobile communication for personal reasons is still a largely unexplored area. (Some of the studies that have provided insight include (Carroll et al., 2001; Ling and Yttri, 1999; Palen et al., 2000).) The second reason is that the mobile phone has become an ubiquitous device, whose initial use at acquisition time might be instrumental, i.e., motivated by specific tasks (either work-related or for use in the coordination of family life), but whose usage pattern quickly becomes in practice an integrated part of life (Ling and Yttri, 1999; Palen et al., 2000), supporting a diverse range of personal communication.

This is especially true of the population that is currently a great consumer of mobile communication, namely teenagers and young adults. This population does not perceive the use mobile phone as instrumental, but rather as a support for social integration, negotiation and networking (Carroll et al, 2001; Ling and Yttri, 1999). Our investigations must therefore also have a special focus on this important population segment.

\section{METHOD DESCRIPTION}

We chose to address our research question using a qualitative method. This is motivated by the fact that, as previously mentioned, very little has been published on the use of mobile phones which would have enabled us to approach the problem with an initial set of hypothesis. Given this lack of substantial and current information, we needed to uncover large trends of usage patterns. A qualitative approach, well suited to the detailed observation of a small number of cases and yielding contextually rich information that can be analysed from a number of perspectives, was therefore in order. 
We interviewed 13 mobile phone owners, each day for a period of a week, asking them to tell us about each of the personal calls or text messages they placed in the last 24 hours. Note that because our study is focused on factors that motivate the choice of the mobile phone, we were only interested in out-going communications, not in in-coming ones. Indeed, users only make the choice to use the mobile phone when they place a call, not when they answer one. We had provided the informants with small, custom-made diaries in which they could record their outgoing communications and which they could carry around with their mobile phone. The role of these mini-diaries was to act as a memory jotter: the idea was for the informants to record enough of the conversation or message to remember it at the time of the interview.

The age range of the informants was between 19 and 33, with an average of 25 . There were 6 females and 7 males. Three were full-time workers and parents and the other 10 were full-time students, living with their parents. Most of the latter were also part-time workers. All the participants, like most people today had busy lifestyles: students all had one or two part-time jobs, workers had families to attend to, and all had extensive social networks.

All the participants were experienced mobile phone users. Indeed, results from (Palen et al, 2000) show that early patterns of use and early perception of the mobile phone differs substantially from stabilized ones. We were interested in stabilized usage patterns.

Each interview gathered the following information:

- the reasons for the communication and its general content,

- the circumstances of the communication: when and from where it was placed, with a special focus on public or private spaces, its degree of urgency, of sensitivity, etc.,

- the background to the communication or its general circumstances e.g., is it part of a regular pattern, was it pre-agreed or expected in some sense, what were the caller's set of motivations, etc.,

- the relationship between the sender and the recipient(s) (in decreasing order of familiarity: family, close friends, distant friends or acquaintances, service or goods provider),

- whether the communication was the only activity performed at the time (the main task), or whether it was performed in conjunction with or in support of another (a secondary task),

- the reasons for choosing the text channel when this was the case. We focused on the reasons for choosing text (as opposed to the reasons for either voice or text), because we quickly realised that voice is perceived by mobile phone users are the default, natural choice, while text messaging is a decision. 
We also asked each informant the following general questions:

- their age,

- their family situation and their occupation (student, working part-time or full time, etc.),

- their experience as regular mobile phone users,

- their initial reason for buying a mobile phone,

- the cost structure for their mobile communications (i.e., their costing plans).

\section{RESULTS AND ANALYSIS}

We collected a total of 138 "stories" about specific mobile communications: 89 about outgoing calls, and 49 about outgoing text messages. As expected given our focus on personal communication, all but 2 instances were of communications with close friends or family.

We analysed the stories we gathered from three perspectives, each of which is discussed in detail in the next sections. First, we examined the reasons invoked by the informants for choosing mobile communication. Secondly we examined the nature of the communication itself - its contents and its intent (or goal). Thirdly, we examine the reasons for using text messaging (as opposed to a phone call).

\subsection{Reasons for choosing a mobile communication device}

Here, we report the reasons participants invoked for choosing to place a communication from their mobile phone, rather communicating in another way (including face-to-face and the use of a landline). The main reasons for using a mobile phone are discussed below (also see Table 1). 


\begin{tabular}{lc}
\hline Reason & \% of communications logged \\
\hline phone functionality & 28.3 \\
cost structure & 28.3 \\
perceived only option & 18.9 \\
travelling & 16.7 \\
spontaneity of communication & 15.2 \\
appropriate for circumstances & 7.2 \\
spontaneity of event & 6.5 \\
urgency / time-criticality & 5.8 \\
fill dead time & 5.1 \\
\hline
\end{tabular}

Table 1. Reasons for choosing a mobile device

Note that any one communication could be motivated by a combination of several reasons.

Phone functionality. A good proportion of calls are made from mobile phones simply because they offer functionality that most land phones do not. Among these, the most cited are the call history, which automatically stores the phone numbers of incoming and outgoing calls, the personalized phone book and the fact that a stored number does not need to be dialled by the phone user. Even in situations where a landline is available and would be cheaper, the mobile phone can be preferred because users can look up a number easily and avoid dialling. Many participants also prefer to call back someone who called their mobile phone from their mobile phone, again because dialling can be done automatically.

Cost structure. The user's costing plan is an important reason for using the mobile phone. For example, under some circumstances (time of day, carrier company of recipient mobile, etc.) calls are free for some period of time (typically 20 minutes). Under other types of costing plans, users pay a fixed amount per month for a fixed allocated use time, and want to utilize this as much as possible (without going over, of course). Maximizing utility for cost is an important factor motivating calls from the mobile phone, including in non-mobile situations (e.g., from home, instead of the landline). Cost is also an important parameter in the choice of sending a text message, especially when the goal of the communication is not specific, but linked to "relationship maintenance" (see below, section 3.2).

Perceived only option. Even when other options are available, such as landlines or public phones, the mobile phone is often perceived as the "only option" because of its ease of access. For example, participants used the mobile phone from their bed, in order to avoid getting up, even if a landline was available in another room. Similarly, participants use the mobile phone while walking on the street, disregarding the option of public phones. 
Travelling. Travelling (including walking, being in a car or public transport) here describes a reason for placing a communication, rather than as a description of the activity that occurred while communicating. To illustrate the difference, a call to finalize the details of a meeting place in order to plan one's route would fall in this category, while a call placed to chat while stuck in traffic would not. It is interesting to note that travelling is not a major reason for using the mobile phone. Even communications placed while travelling (i.e., travelling as a description of the circumstances of the call rather than as its reason) only make-up a minority of the communications we gathered.

Spontaneity of communicating. This category includes communications that had to be placed and were placed as soon as the participant thought of it. (It differs from the "spontaneity of event" category, described next, which includes communication to spontaneously arrange a meeting or event. Here, we refer to communications that had to be placed anyway.) The mobile phone allows users to avoid remembering to call and planning their communications in "batches".

Appropriateness for circumstances. We gathered several instances of a group sitting in a restaurant or café and deciding to contact a common friend who is not present, either to invite him or her or to just keep in touch. Here, the mobile phone enables seizing the opportunity as it presented itself.

Spontaneity of event. The mobile phone allows users to have unplanned encounters with friends (e.g., "I'm at the university coffee shop, come and meet me here"). This is valid for both text messaging and phone calls.

Urgency, time criticality. A small number of instances involved urgent or time-critical communications. The mobile phone enables communication in a reactive and timely manner. While issues of safety and urgency may initially motivate the acquisition of a mobile phone (Palen et al, 2000), they only accounted for a small proportion of actual use in stabilized practice in our study.

To fill dead time. Many calls are placed because "I was bored and decided to call a friend". This is typically the case on public transport, during a boring lecture or seminar, or while stuck in traffic. Sometime calling or sending a text message at a "dead time" is planned by the user, e.g., "I thought I would "text" him from the airport because I knew I would have time to kill there." The calls or messages which were placed in such "dead times" were mostly of the "relationship maintenance" type (see below).

These results show that the reasons why people use mobile phones are far from being only linked to mobility. As shown above, there are numerous reasons that motivate people to use their mobile phones, even when a landline is available. 
It is also worth noting that teenagers and young adults often use the mobile phone as a personal line (instead of having a second home phone with a different number). Pragmatically, this type of arrangement keeps individual communication bills separate from household bills, and the mobile phone is viewed as a landline with additional capabilities and convenience. But there is also a social factor at play here. For people in this age range, social networking is vital and contributes in an important way to self-identity. Thus social communication is viewed as a highly personal activity. Having a personal, exclusive communication device re-enforces this feeling. (See (Carroll et al., 2001) for the role of the mobile phone on the formation of identity and a sense of belonging.)

Cost is an important consideration in the use of the mobile phone, and it plays a dual role: it can act as a motivator, for example when calls to a mobile phone are cheaper if placed from a mobile than if place from landline, but can also act as a barrier: several of the participants complained that they had problems controlling the costs of their mobile communications - use of the mobile phone is described as "addictive".

Finally, it seems many of the reasons invoked for using a mobile phone (as opposed to another communication device, or to face-to-face communication when it is possible) revolve around its enhanced capabilities as compared to a landline (e.g., the "functionality" category above), its ease of access (e.g. "perceived only option", "urgency", "fill dead time") and its flexibility and timeliness (e.g. "spontaneity of communication", "spontaneity of event", "appropriate for circumstances"). These attributes are especially well suited to the requirements of our participants for a "fluid" lifestyle. By "fluid", we mean flexible, reactive to changing circumstances with minimum effort.

\subsection{Intents of mobile communication}

We now turn to the intents or goals of the communications we gathered. We found two broad types of communications from a mobile phone: relationship maintenance and coordination of events. They are discussed here, and summarised in Table 2. (Note that the same communication could have several distinct intents.) 


\begin{tabular}{cc}
\hline Intent & $\begin{array}{c}\text { \% of communications } \\
\text { logged }\end{array}$ \\
\hline Planning and coordination & 71.1 \\
organise a meeting or event & 26.1 \\
"plastic" plan & 19.6 \\
changes to firm plans & 18.1 \\
initiation of a regular plan & 5.1 \\
co-ordinate resource / task & 2.2 \\
sharing & \\
Relationship maintenance & 27.5 \\
Others - Miscellaneous & 15.9 \\
\hline
\end{tabular}

Table 2. Intents of mobile communication

A substantial proportion of the communications we logged concerned expressions of feelings, keeping in touch with friends, informing a partner of one's whereabouts, or simply chitchats. We refer to this type of communication as relationship maintenance. The use of the mobile phone for relationship maintenance is very diverse. It ranges from short text messages (e.g., one word to a girlfriend), to regular 20 minutes chats at times when the use of the mobile phone is free.

One type of relationship maintenance communications concerned what we term "loose keeping in touch". "Loose keeping in touch" concerns the general expression of friendship or love with no sense of urgency or timeliness - for example, daily long chitchats with a boyfriend or girlfriend. Some of these communications may also be short "drops" that do not require interactivity and hence are especially well suited to text messaging (e.g., "boring lecture, thinking of you"). For example, one participant told us she often "texts" people she sees every day just to say hello. Other examples include keeping friends and relatives informed of significant events in one's life (e.g. "just bought a new bike" or "got a good mark on my exam").

In this regard, we found that there seems to be an emerging "etiquette" for choosing the appropriate channel for "loose keeping in touch" communications: the channel chosen seems to depend on the closeness of the relationship between the conversing parties. For example, to express good wishes (for a birthday, travel, etc.) to close friends, our participants would call, while they would favour sending a message to distant friends.

Another type of relationship maintenance communication we gathered concerned more pressing or compelling motivations. In these cases, calling is chosen over "texting", probably because voice communication is "richer" than text (it has more cues, it conveys presence better) and because interactivity is important. Let us report two revealing examples of this. In 
the first instance, a student phoned her boyfriend before entering an exam room because "I was nervous and talking to him calms me down". In the second instance, a young child was disturbed by that fact that her mother was away at work one afternoon, and asked her father to ring up Mom from the day-care "just to hear her voice". In these cases, emotional support and reassurance were sought, and vocal mobile communication was appropriate in addressing these needs in a flexible, time-sensitive way, regardless of location.

The other large category of intent concerned the coordination of actions and events, especially of meetings and rendezvous. This has been referred to elsewhere as the micro-coordination of life events (Ling and Yttri, 1999). There are several types of coordination which have emerged from our study, and which seemed typical of mobile communication. They are described below.

Organising an event. An important proportion of the communications we logged were placed to organize meetings or event (of other types than "plastic planning" - see below). Text messaging is frequently used for organizing group events since users can take advantage of the "group-send" functionality. In these cases, the communication is terse, resting on a good deal of common knowledge among the parties.

The "plastic plan". We borrow the term "plastic plan" from one of our participants to refer to a plan that is only sketched when first established, and gradually refined over several communications. Access to mobile communication allows people to have initially vague plans (e.g., "Meet me for lunch in the city"), which are refined gradually or on the spot (e.g., "I'm on my way to the city now, meet you in half an hour at Town Hall."). "Plastic planning" definitely constitutes a strongly emerging way of coordinating social rendezvousing. This finding echoes the "softening of time" referred to in (Ling and Yttri, 1999). One participant told us that the reason he acquired a mobile phone was that he missed several rendezvous' with his friends, all mobile phone users, because he could not be reached at the time of "firming up the plan". Plastic planning allows flexibility and reactivity, and thus is well suited to the lifestyle of the young population.

Changes to firm plans. This especially concerns notifications of delays when a firm plan was made, but unexpected events prevent it from happening as planned (e.g., traffic delays). Other instances are change of plans (e.g., "I've changed my mind about the lift, have you left the parking lot yet?"), and people losing each other in a crowd (e.g., "I've lost you, where are you?").

Initiation of a regular plan. Text messaging is used to trigger or confirm regular meetings with minimum communication content. Again, this type of message relies heavily on implicit, shared knowledge among the 
participants, hence its brevity. For example, the message "meet me before the lecture" is understood to mean "meet me at the university café, 15 minutes before the lecture and bring your lecture notes so I can photocopy them". The place, precise time and purpose of the rendezvous are known to the participants through previous shared experiences, and therefore don't need to be specified. The communication serves as either a trigger or a confirmation of a regular plan.

Coordinate resource or task sharing. This concerns for example coordinating the sharing of the household car in a flexible and time-sensitive way. Tasks also are distributed in this way (e.g., "I'll pick up food on the way home")

In synthesis, most communications with mobile phones pertain to relationship maintenance or to coordination. "Plastic planning", which. allows for fluid social coordination and can only be possible with a mobile phone, seems to be a growing phenomenon, as it is especially well suited to the life style of the young population. These observations indicate that the mobile phone is an essential element supporting the elaboration and maintenance of social life for our participants.

\subsection{Reasons for text messaging}

Note that while land phones (private or public) are competing devices to mobile phones, there is no competitor to text messaging. (Email and instant messaging are far from being the stationary equivalent to text messaging, as they are very different in terms of overheads, accessibility, length and complexity of messages, functionality, etc. They are perceived as other mediums altogether.) Still, sending a text message is generally perceived as the secondary use of the mobile phone, while phoning is its primary use, the "default" option. Consequently, sending a text message require an actual choice from our participants (contrary to phoning).

Below are the characteristics of text messaging which were specifically invoked as motivations for its use (see also Table 3). 


\begin{tabular}{lc}
\hline Reason & $\begin{array}{c}\% \text { of textual communications } \\
\text { logged }\end{array}$ \\
\hline recipient friendly & 36.7 \\
avoid conversation & 28.6 \\
channel consistency & 20.4 \\
non-instrumental info. drop & 20.4 \\
socially acceptable to text & 12.2 \\
cost vs. calling & 8.2 \\
fall back option & 8.2 \\
"send to group" functionality & 6.1 \\
\hline
\end{tabular}

Table 3. Reasons for text messaging

Recipient-friendly. Because it is not interactive, text messaging is perceived as more "recipient-friendly" than calling: it leaves the recipient in charge of when to take the communication, and whether to answer it or not. It has been described by our participants as more "discreet" and more "appropriate" than calling, especially when the recipient is thought to be unavailable (e.g., busy or sleeping). Hence, non urgent communications are often transmitted through text rather than voice.

Avoid conversation. Our participants often send a message when they do not want to engage in conversation but still want to communicate. In many cases, this may be for the sake of time or efficiency. Here, text messaging seems to have the equivalent function to calling someone's vocal mailbox. In addition, probably because it is not interactive, messaging is also perceived as providing a "social distance" relatively to a phone call. As mentioned above, this is considered appropriate when the communicating parties are not in a close relationship.

Channel consistency. An important number of text messages were sent because "I was answering a previous text message so I didn't want to call". Text messaging is often used in response to an in-coming text message. This refers to a phenomenon that may be termed "channel consistency", whereby someone prefers to return a communication in the same channel as it was received. Reasons behind this may involve a sense of social appropriateness: if the initiator of a communication has chosen one channel, then it is considered appropriate that follow-up communications should be made using that same channel.

Non-instrumental information drops. Text messaging is also used for one-way "non-instrumental" information drops, i.e., for communications that are merely informative, not urgent, and do not specifically contribute to a task (e.g., "got a good mark on my exam"). There is often a pre-existing arrangement, implicit or explicit, that information will be sent, but this is not 
necessarily the case. Again, text messaging is preferred here because no interaction with the interlocutor is required.

Socially acceptable to text. For the sender, text messaging is also more discreet and, not being interactive, less demanding in terms of attention. It is used in "public" situations, or in situations where talking on the phone would not be socially appropriate: during a lecture, on busy public transport or in small group settings without isolating oneself from the conversation. For example, we encountered instances of participants sending text messages while with a group of friends - sending a message allowed them to continue interacting with the group, while isolating one self to place a phone call would have been perceived as rude to the group.

Cost compared to calling. Text messaging is also used as the cheaper alternative to calling. We see again that cost is a consideration for mobile phone users, but, surprisingly given that "texting" is considerably cheaper than calling, it did not emerge as a major factor for our participants.

Fall back option. In a minority of cases, a text message is sent when calling fails. Here, reasons might be that the recipient's mobile phone has poor reception or is switched off.

Group communication. Text messaging is used to communicate to a group in a succinct way, taking full advantage of abbreviations and preestablished codes. Here, users take advantage of the "send to group" functionality of the phone.

In conclusion, it is interesting to note that text messaging is mostly used as a communication channel in its own right, not merely as a fall back option. In addition, the use of text messaging seems to be guided by emerging social conventions: in some circumstances, it is judged more appropriate either for the sender or the recipient, than calling. Finally, and contrary to the general findings regarding the use of the mobile phone (section 3.1), text messaging is only marginally motivated by phone functionality (e.g. the "group send") and costs.

\section{CONCLUSION: WHAT HAVE WE LEARNED AND HOW IS IT USEFUL?}

The various factors which were uncovered by this study as motivating the use of the mobile phone, such as its functionality, its cost, its ease of access and its flexibility, as well as the types of communications that are placed from a mobile phone (coordination and relationship maintenance) all contribute to support what we have termed "fluid" social interaction and coordination. The mobile phone is not exclusively used in mobile situations. 
Indeed, its use is much larger than strictly linked to one set of circumstances: it has a major role in supporting today's lifestyles.

Another interesting results of this survey is the fact that the use of the mobile phone is governed by an emerging "mobility etiquette", which, albeit probably still in formation, has nonetheless a definite influence in how and when to communicate. This is especially true for text messaging.

Thirdly, findings pertaining to the use of text messaging show that it is considered as a medium in its own right, not only as a fall back option.

Understanding the practices around the use of a particular technology is useful in many ways. Firstly, this understanding helps to design new enhancements to that technology. For example, mobile phone companies have announced that they will shortly release a miniature camera to be hooked on the mobile phone, thereby adding video communication, synchronous and asynchronous, to the two channels (text and voice) currently available to the mobile phone user. Undoubtedly, the uptake of the "mobile videophone" will be rooted in the current practices of mobile phone users. Understanding the latter can therefore give clues to forecast the former, and thus help in the definition and positioning of mobile video communication.

Another benefit of understanding user practices is to add to current knowledge regarding technology adoption. The investigation presented here is an exploration into the factors that lead to voluntary technology adoption (notwithstanding the social factors in play) for "recreational" or personal purposes, (i.e., not work-related). We have not referred, in this study, to theoretical frameworks of technology adoption or acceptance, for example as those defined in (Davis, 1993; Rogers, 1995), because these frameworks pertain to the work place and to technologies that support work functions. We submit that "recreational" technologies are perceived, assessed and used in ways that are very different to work-related ones. Hence, a theory of technology adoption for personal use still needs to be built, based on case studies such as this one.

\section{ACKNOWLEDGEMENTS}

Many thanks to Robert Tot, from CSIRO/CMIS, for his participation to this study. 


\section{REFERENCES}

Carroll, J., Howard, S., Vetere, F., Peck, J. \& Murphy, J. (2001), Identity, Power and Fragmentation in Cyberspace: Technology Appropriation by the Young People. Working Paper 01/IDG/2001, Dept. of Information Systems, University of Melbourne, Australia. Available at http://www.dis.unimelb.edu.au/staff/idgroup/workingpapers.htm

Davis, F.D. (1993), User acceptance of information technology: system characteristics, user perceptions and behavioral impacts, International Journal of Man-Machine Studies, Vol. 38, No. 3, pp. 475-487.

Lenhart, A., Rainie, L., Lewis, O. (2001), Teenage Life Online: The rise of the instantmessage generation and the Internet's impact on friendships and family relationships. Pew Internet Project report, June. http://www.pewinternet.org/reports/toc.asp?Report=36

Ling, R. \& Yttri, B. (1999), Nobody sits at home and waits for the telephone to ring: Micro and hyper-coordination through the use of the mobile telephone. Telenor R\&D report 30/99. ISBN 82-423-0505-6.

Palen, L. Salzman, M. \& Young, E. (2000), Going Wireless: Behavior and Practice of New Mobile Phone Users. Proceedings of the Computer Supported Collaborative Work (CSCW)'00 Conference (Philadelphia, Pennsylvania Dec.), ACM Press, pp. 201-210.

Rogers, Everett, M. (1995), Diffusion of Innovations. Free Press, New York, (fourth edition). 